\title{
SINGULAR CASE OF GENERALIZED FIBONACCI AND LUCAS MATRICES
}

\author{
Marko Miladinović and Predrag Stanimirović
}

\begin{abstract}
The notion of the generalized Fibonacci matrix $\mathcal{F}_{n}^{(a, b, s)}$ of type $s$, whose nonzero elements are generalized Fibonacci numbers, is introduced in the paper [23]. Regular case $s=0$ is investigated in [23]. In the present article we consider singular case $s=-1$. Pseudoinverse of the generalized Fibonacci matrix $\mathcal{F}_{n}^{(a, b,-1)}$ is derived. Correlations between the matrix $\mathcal{F}_{n}^{(a, b,-1)}$ and the Pascal matrices are considered. Some combinatorial identities involving generalized Fibonacci numbers are derived. A class of test matrices for computing the Moore-Penrose inverse is presented in the last section.
\end{abstract}

\section{Introduction}

Let $\mathbb{C}$ be the set of complex numbers, $\mathbb{C}^{m \times n}$ the set of $m \times n$ complex matrices, and $\mathbb{C}_{r}^{m \times n}$ a subset of $\mathbb{C}^{m \times n}$ consisting matrices of rank $r$ : $\mathbb{C}_{r}^{m \times n}=$ $\left\{X \in \mathbb{C}^{m \times n} \mid \operatorname{rank}(X)=r\right\}$. By $A^{\dagger}$ we denote the Moore-Penrose inverse of $A$, i.e., the unique matrix $A^{\dagger}$ satisfying [4]

$$
A A^{\dagger} A=A, A^{\dagger} A A^{\dagger}=A^{\dagger},\left(A A^{\dagger}\right)^{*}=A A^{\dagger},\left(A^{\dagger} A\right)^{*}=A^{\dagger} A,
$$

where the superscript $*$ denotes conjugate and transpose: $A^{*}=\bar{A}^{T}$.

There are well known various methods for computing the Moore-Penrose inverse (see for example [4], [26]). The most commonly implemented method in programming languages is the Singular Value Decomposition (SVD) method, that is implemented, for example, in the "pinv" function from Matlab, as well as in the standard MATHEMATICA function "PseudoInverse" [8], [28]. This method is very accurate, but time consuming when the matrix is large [4], [8]. Other well-known methods are Greville's algorithm, the full rank QR factorization by Gram-Schmidt orthonormalization (GSO), and iterative methods of various orders [4].

Received February 3, 2009.

2010 Mathematics Subject Classification. 05A10, 11B39, 15A09.

Key words and phrases. generalized Fibonaci numbers, generalized Fibonaci matrix, Lucas numbers, Lucas matrix. 
Grevile in [11] proposed a recursive algorithm which relates the MoorePenrose pseudoinverse of a matrix $R$ augmented by an appropriate vector $r$ with the pseudoinverse $R^{\dagger}$ of $R$. A generalization of this statement, which is applicable to rational matrices and its implementation in the package MATHEMATICA is presented in [24]. In the present paper we use this implementation in the pseudoinverse computation.

The Fibonacci numbers $\left\{F_{n}\right\}_{n=0}^{\infty}$ are the terms of the sequence $0,1,1,2,3,5$, $\ldots$, where each term is the sum of the two preceding terms, and we get things started with 0 and 1 as $F_{0}$ and $F_{1}$. You cannot go very far in the lore of Fibonacci numbers without encountering the companion sequence of Lucas numbers $\left\{L_{n}\right\}_{n=0}^{\infty}$, which follows the same recursive pattern as the Fibonacci numbers, but begins with $L_{0}=2$ and $L_{1}=1$. The sequence of Lucas numbers is therefore $2,1,3,4,7, \ldots$ We also observe so-called generalized Fibonacci numbers, $\left\{F_{n}^{(a, b)}\right\}_{n=0}^{\infty}$, which satisfy the same recursive formula $F_{n+2}^{(a, b)}=F_{n+1}^{(a, b)}+$ $F_{n}^{(a, b)}, n=0,1, \ldots$, but starting with arbitrary initial values $F_{0}^{(a, b)}=a$ and $F_{1}^{(a, b)}=b,($ see for example [18], [16], [25], [19, Chapter 7]).

The $n \times n$ Fibonacci matrix $\mathcal{F}_{n}=\left[f_{i, j}\right](i, j=1, \ldots, n)$ is defined by [20]:

$$
f_{i, j}= \begin{cases}F_{i-j+1}, & i-j+1 \geq 0, \\ 0, & i-j+1<0 .\end{cases}
$$

The inverse and Cholesky factorization of the Fibonacci matrix are given in [20]. The relations between the Pascal matrix and the Fibonacci matrix are studied in [21].

As an analogy of the Fibonacci matrix, the $n \times n$ Lucas matrix $\mathcal{L}_{n}=$ $\left[l_{i, j}\right](i, j=1, \ldots, n)$ is defined in [32]:

$$
l_{i, j}= \begin{cases}L_{i-j+1}, & i-j \geq 0, \\ 0, & i-j<0 .\end{cases}
$$

In the paper [22] the author investigated the inverse and Cholesky factorization of the matrix $\mathcal{U}_{n}$ with entries

$$
u_{i, j}= \begin{cases}U_{i-j+1}, & i-j+1 \geq 0, \\ 0, & i-j+1<0,\end{cases}
$$

where $U_{n}$ is the non-degenerated second order sequence $U_{n+1}=A U_{n}+B U_{n-1}$, $\delta=\sqrt{A^{2}+4 B}$ real, and where $A, B, U_{1}$ are integers and $U_{0}=0$ (i.e., $A=B$ ). In $[22]$ the author also generalized these results to $r$-order recurrent sequence satisfying $U_{0}=U_{-1}=\cdots=U_{2-r}=0, U_{1}$ arbitrary.

Notions of Fibonacci and Lucas matrix are included in the following definition from [23].

Definition 1.1. Let $F_{n}^{(a, b)}$ be the $n$-th generalized Fibonacci number, where the starting members of the Fibonacci array are $F_{0}=a$ and $F_{1}=b$, and 
where $a, b \in \mathbb{C}$. The generalized Fibonacci matrix of type $s$ and of the order $n$, denoted by $\mathcal{F}_{n}^{(a, b, s)}=\left[f_{i j}^{(a, b, s)}\right]$, is defined by

$$
f_{i, j}^{(a, b, s)}=\left\{\begin{array}{ll}
F_{i-j+1}^{(a, b)}, & i-j+s \geq 0 \\
0, & i-j+s<0
\end{array} \quad i, j=1, \ldots, n .\right.
$$

Example 1.1. The $6 \times 6$ generalized Fibonacci matrix of type -1 is equal to

$$
\mathcal{F}_{6}^{(a, b,-1)}=\left(\begin{array}{cccccc}
0 & 0 & 0 & 0 & 0 & 0 \\
a+b & 0 & 0 & 0 & 0 & 0 \\
a+2 b & a+b & 0 & 0 & 0 & 0 \\
2 a+3 b & a+2 b & a+b & 0 & 0 & 0 \\
3 a+5 b & 2 a+3 b & a+2 b & a+b & 0 & 0 \\
5 a+8 b & 3 a+5 b & 2 a+3 b & a+2 b & a+b & 0
\end{array}\right) .
$$

We observe that $\mathcal{F}_{n}^{(a, b,-1)}$ is a strictly lower triangular Toeplitz matrix, and therefore it is singular. Toeplitz matrices are matrices having constant entries along their diagonals. This structure is very interesting in itself for all the rich theoretical properties which it involves, but at the same time it is important for the dramatic impact that it has in applications. Toeplitz matrix arises in scientific computing and engineering, for example, image processing, numerical differential equations and integral equations, time series analysis and control theory (see, for example [6], [17]). Toeplitz matrices arise quite naturally in the study of discrete time random processes. Covariance matrices of weakly stationary processes are Toeplitz and triangular Toeplitz matrices provide a matrix representation of causal linear time invariant filters [10]. Toeplitz matrices are also closely connected with Fourier series, because the multiplication operator by a trigonometric polynomial, compressed to a finite-dimensional space, can be represented by such a matrix. In communication theory, a finite duration impulse response (FIR) filter in discrete-time is constructed for purposes of linear prediction of a random process $X(t)$. The autocorrelation matrix of $X$ is found to be a Toeplitz matrix. Also, textbooks go one step further in trying to find the optimal predictor coefficients, by taking the inverse of this matrix. On the other hand, in singular cases, there are a number of papers which investigate the usual inverse and various generalized inverses of some Toeplitz-like matrices. The generalized inverse for Hankel and Toeplitz matrices can be found in $[1,9,14,15,13,27,29]$. Hartwig and Shoaf [12] considered the group inverse and the Drazin inverse of singular bidiagonal and triangular Toeplitz matrices.

We compute the pseudoinverse of generalized Fibonacci matrix $\mathcal{F}_{n}^{(a, b,-1)}$, of type -1 , whose nonzero elements are generalized Fibonacci numbers $F_{n}^{(a, b)}$. Results obtained in [23] include known facts about the generalized Fibonacci matrices and their properties only in regular cases $s=0$ and $s=1$. At this moment we consider matrices $\mathcal{F}_{n}^{(a, b, s)}, s<0$ with a special view on $s=-1$. A correlation between the generalized Fibonacci matrix $\mathcal{F}_{n}^{(a, b,-1)}$ and the Pascal 
matrix of the first and the second kind is considered in Section 3. An application of these results is reached in the fourth section, where some combinatorial identities involving generalized Fibonacci numbers and binomial coefficients are derived. In the last section we get a class of test matrices for computing the Moore-Penrose inverse.

\section{Generalized Fibonacci matrix of type $s=-1$ and its inverse}

In this section we compute the Moore-Penrose inverse for singular generalized Fibonacci matrix $\mathcal{F}_{n}^{(a, b,-1)}$.

Lemma 2.1. For the generalized Fibonacci numbers $F_{n}^{(a, b)},(a \neq-b)$ and for two arbitrary integers $i, j$ satisfying $i \geq j+2$ the following is valid:

$$
\sum_{k=j+1}^{i-1} F_{i-k+1}^{(a, b)} \frac{\left(a^{2}+a b-b^{2}\right)(-b)^{k-j-1}}{(a+b)^{k-j+2}}=-\frac{b}{(a+b)^{2}} F_{i-j+1}^{(a, b)}+\frac{1}{(a+b)} F_{i-j}^{(a, b)} .
$$

Proof. It is not difficult to verify the following generalization of the Binet's Fibonacci number formula (see [23]):

$$
F_{n}^{(a, b)}=c_{1} \alpha^{n}+c_{2} \beta^{n},
$$

where

$$
\begin{aligned}
c_{1} & =\frac{5 a+\sqrt{5} a-2 \sqrt{5} b}{10}, c_{2}=\frac{5 a-\sqrt{5} a+2 \sqrt{5} b}{10}, \\
\alpha & =\frac{1-\sqrt{5}}{2}, \quad \beta=\frac{1+\sqrt{5}}{2} .
\end{aligned}
$$

From previous equalities we have

$$
\alpha \beta=-1, \alpha+\beta=1, \alpha-\beta=-\sqrt{5} .
$$

Let us denote

$$
I=\sum_{k=j+1}^{i-1} F_{i-k+1} \frac{(-b)^{k-j-1}}{(a+b)^{k-j+2}} .
$$

By applying (2.2) and simple transformations, we obtain the following:

$$
\begin{aligned}
I & =\sum_{k=j+1}^{i-1}\left(c_{1} \alpha^{i-k+1}+c_{2} \beta^{i-k+1}\right) \frac{(-b)^{k-j-1}}{(a+b)^{k-j+2}} \\
& =\frac{c_{1} \alpha^{2}(a+b)^{j-i-1}}{(-b)^{j-i+2}} \sum_{k=j+1}^{i-1}\left(\frac{-\alpha(a+b)}{b}\right)^{i-k-1}+\frac{c_{2} \beta^{2}(a+b)^{j-i-1}}{(-b)^{j-i+2}} \sum_{k=j+1}^{i-1}\left(\frac{-\beta(a+b)}{b}\right)^{i-k-1} \\
& =\frac{c_{1} \alpha^{2}(a+b)^{j-i-1}}{(-b)^{j-i+2}} \cdot \frac{1-\left(-\frac{\alpha(a+b)}{b}\right)^{i-j-1}}{1+\frac{\alpha(a+b)}{b}}+\frac{c_{2} \beta^{2}(a+b)^{j-i-1}}{(-b)^{j-i+2}} \cdot \frac{1-\left(-\frac{\beta(a+b)}{b}\right)^{i-j-1}}{1+\frac{\beta(a+b)}{b}} .
\end{aligned}
$$


After some algebraic transformations we get

$$
\begin{aligned}
I= & \frac{c_{1} \alpha^{2}(a+b)^{j-i-1}(-b)^{i-j}+c_{1} \alpha^{i-j+1} \frac{b}{(a+b)^{2}}}{(b+\alpha a+\alpha b)(b+\beta a+\beta b)} \\
& +\frac{-c_{1} \alpha^{2} \beta(a+b)^{j-i}(-b)^{i-j-1}+c_{1} \alpha^{i-j+1} \frac{\beta}{(a+b)}}{(b+\alpha a+\alpha b)(b+\beta a+\beta b)} \\
& +\frac{c_{2} \beta^{2}(a+b)^{j-i-1}(-b)^{i-j}+c_{2} \beta^{i-j+1} \frac{b}{(a+b)^{2}}}{(b+\alpha a+\alpha b)(b+\beta a+\beta b)} \\
& +\frac{-c_{2} \beta^{2} \alpha(a+b)^{j-i}(-b)^{i-j-1}+c_{2} \beta^{i-j+1} \frac{\alpha}{(a+b)}}{(b+\alpha a+\alpha b)(b+\beta a+\beta b)} .
\end{aligned}
$$

Using identities $(b+\alpha a+\alpha b)(b+\beta a+\beta b)=-\left(a^{2}+a b-b^{2}\right),(2.3)$ and grouping similar members using (2.2) we have that the following is valid:

$$
\begin{aligned}
I & =\frac{(a+b)^{j-i-1}(-b)^{i-j} F_{2}^{(a, b)}+\frac{b}{(a+b)^{2}} F_{i-j+1}^{(a, b)}+(a+b)^{j-i}(-b)^{i-j-1} F_{1}^{(a, b)}-\frac{1}{a+b} F_{i-j}^{(a, b)}}{-\left(a^{2}+a b-b^{2}\right)} \\
& =\frac{-\frac{b}{(a+b)^{2}} F_{i-j+1}^{(a, b)}+\frac{1}{a+b} F_{i-j}^{(a, b)}}{\left(a^{2}+a b-b^{2}\right)} .
\end{aligned}
$$

Finally, from

$$
\sum_{k=j+1}^{i-1} F_{i-k+1}^{(a, b)} \frac{\left(a^{2}+a b-b^{2}\right)(-b)^{k-j-1}}{(a+b)^{k-j+2}}=\left(a^{2}+a b-b^{2}\right) I
$$

we complete the proof.

Lemma 2.2. For $n \times n$ matrix $\mathcal{X}_{n}^{(a, b)}=\left[x_{i, j}\right]$ defined by

$$
x_{i, j}= \begin{cases}-(-b)^{i-j-1} \cdot \frac{\left(a^{2}+a b-b^{2}\right)}{(a+b)^{i-j+2}}, & (i>j, i \neq n, j \neq 1) \\ -\frac{a+2 b}{(a+b)^{2}}, & (i=j, i \notin\{1, n\}) \\ \frac{1}{a+b}, & i+1=j \\ 0, & \text { otherwise, }\end{cases}
$$

the following is valid

$$
\mathcal{F}_{n}^{(a, b,-1)} \cdot \mathcal{X}_{n}^{(a, b)}=\mathcal{V}_{n}
$$

where $\mathcal{V}_{n}=\left[v_{i, j}\right]$ is $n \times n$ matrix given by

$$
v_{i, j}= \begin{cases}1, & (i=j, i \neq 1) \\ 0, & \text { otherwise. }\end{cases}
$$

Proof. Let $\sum_{k=1}^{n} f_{i, k}^{(a, b,-1)} x_{k, j}=v_{i, j}$. First we want to prove that $v_{i, j}=0$, $i \neq j$. Obviously $v_{i, j}=0, i<j$.

In the case $i=j+1$ one can verify the following:

$$
v_{j+1, j}=\frac{1}{a+b} f_{j+1, j-1}-\frac{a+2 b}{(a+b)^{2}} f_{j+1, j}
$$




$$
\begin{aligned}
& =\frac{1}{a+b} F_{3}^{(a, b)}-\frac{a+2 b}{(a+b)^{2}} F_{2}^{(a, b)} \\
& =\frac{a+2 b}{(a+b)}-\frac{a+2 b}{(a+b)} \\
& =0 .
\end{aligned}
$$

When $i>j+1$ applying the results from Lemma 2.1 we obtain

$$
\begin{aligned}
v_{i, j} & =\frac{1}{a+b} f_{i, j-1}-\frac{a+2 b}{(a+b)^{2}} f_{i, j}-\sum_{k=j+1}^{i-1} f_{i, k} \frac{\left(a^{2}+a b-b^{2}\right)(-b)^{k-j-1}}{(a+b)^{k-j+2}} \\
& =\frac{1}{a+b} F_{i-j+2}^{(a, b)}-\frac{a+2 b}{(a+b)^{2}} F_{i-j+1}^{(a, b)}+\frac{b}{(a+b)^{2}} F_{i-j+1}^{(a, b)}-\frac{1}{(a+b)} F_{i-j}^{(a, b)} \\
& =\frac{1}{a+b} F_{i-j+2}^{(a, b)}-\frac{1}{a+b} F_{i-j+1}^{(a, b)}-\frac{1}{a+b} F_{i-j}^{(a, b)} \\
& =0 .
\end{aligned}
$$

Finally, for $i=j>1$ we have

$$
v_{i, i}=f_{i, i-1} \cdot x_{i-1, i}=\frac{1}{a+b} F_{2}^{(a, b)}=\frac{a+b}{a+b}=1 .
$$

It is obvious that $v_{1,1}=0$, and the proof is completed.

Lemma 2.3. Let $\mathcal{X}_{n}^{(a, b)}=\left[x_{i, j}\right]$ be $n \times n$ matrix defined by (2.4). The following holds

$$
\mathcal{X}_{n}^{(a, b)} \cdot \mathcal{F}_{n}^{(a, b,-1)}=\mathcal{Z}_{n}
$$

where $\mathcal{Z}_{n}=\left[z_{i, j}\right]$ is $n \times n$ matrix given by

$$
z_{i, j}= \begin{cases}1, & (i=j, i \neq n) \\ 0, & \text { otherwise. }\end{cases}
$$

Proof. The proof can be accomplished in the same way as in previous lemma.

Example 2.1. The $6 \times 6$ matrices $\mathcal{V}_{6}$ and $\mathcal{Z}_{6}$ are equal to

$$
\mathcal{V}_{6}=\left(\begin{array}{cccccc}
0 & 0 & 0 & 0 & 0 & 0 \\
0 & 1 & 0 & 0 & 0 & 0 \\
0 & 0 & 1 & 0 & 0 & 0 \\
0 & 0 & 0 & 1 & 0 & 0 \\
0 & 0 & 0 & 0 & 1 & 0 \\
0 & 0 & 0 & 0 & 0 & 1
\end{array}\right), \quad \mathcal{Z}_{6}=\left(\begin{array}{cccccc}
1 & 0 & 0 & 0 & 0 & 0 \\
0 & 1 & 0 & 0 & 0 & 0 \\
0 & 0 & 1 & 0 & 0 & 0 \\
0 & 0 & 0 & 1 & 0 & 0 \\
0 & 0 & 0 & 0 & 1 & 0 \\
0 & 0 & 0 & 0 & 0 & 0
\end{array}\right)
$$

Theorem 2.1. The $n \times n$ matrix $\mathcal{X}_{n}$ defined by (2.4) is the Moore-Penrose pseudoinverse of the matrix $\mathcal{F}_{n}^{(a, b,-1)}$. 
Proof. Let us denote $\mathcal{F}_{n}=\mathcal{F}_{n}^{(a, b,-1)}, \mathcal{X}_{n}=\mathcal{X}_{n}{ }^{(a, b,-1)}$. Applying results obtained in Lemma 2.2 it is easy to see that

$$
\mathcal{F}_{n} \mathcal{X}_{n} \mathcal{F}_{n}=\mathcal{V}_{n} \mathcal{F}_{n}=\mathcal{F}_{n}
$$

In the same way using the equality (2.6) from Lemma 2.3 we get

$$
\mathcal{X}_{n} \mathcal{F}_{n} \mathcal{X}_{n}=\mathcal{Z}_{n} \mathcal{X}_{n}=\mathcal{X}_{n}
$$

The proof can be easily completed verifying remaining two equations from the Moore-Penrose inverse definition, using simple representations of matrices $\mathcal{V}_{n}$ and $\mathcal{Z}_{n}$ and the fact that they are identical with their conjugate-transpose matrices.

In the remaining of the paper we use the following notation $\mathcal{F}_{n}^{\dagger}(a, b,-1)=$ $\left[f_{i, j}^{\dagger(a, b,-1)}\right]$ for the matrix $\mathcal{X}_{n}^{(a, b,-1)}$.

\section{Generalized Fibonacci matrix and Pascal matrices}

Various types of Pascal matrices $P_{n}$ are investigated in [2], [3], [5], [7], [30], [31]. In this section we want to investigate correlation between the matrix $\mathcal{F}_{n}^{(a, b,-1)}$ and Pascal matrices. Since $\operatorname{rank}\left(\mathcal{F}_{n}^{(a, b,-1)}\right)=n-1$ and $\operatorname{rank}\left(P_{n}\right)=n$, it is not possible to use $P_{n}$ in the usual manner as in [23], [32], [21]. For this purpose we introduce the following definition of Pascal matrices of type $s$.

Definition 3.1. The generalized Pascal matrix of the first kind and of the type $s$, denoted by $\mathcal{P}_{n}^{(s)}[x]=\left[p_{i, j}^{(s)}[x]\right], i, j=1, \ldots, n$ is defined by:

$$
p_{i, j}^{(s)}[x]= \begin{cases}x^{i-j}\left(\begin{array}{c}
i-1 \\
j-1
\end{array}\right), & i-j+s \geq 0, \\
0, & i-j+s<0 .\end{cases}
$$

In the case $x=1$ the generalized Pascal matrix of the first kind and of the type $s$ reduces to Pascal matrix of type $s$, denoted by $\mathcal{P}_{n}^{(s)}=\left[p_{i, j}^{(s)}\right], i, j=1, \ldots, n$, and defined as:

$$
p_{i, j}^{(s)}= \begin{cases}\left(\begin{array}{c}
i-1 \\
j-1
\end{array}\right), & i-j+s \geq 0 \\
0, & i-j+s<0\end{cases}
$$

In the following theorem we define matrix $\mathcal{G}_{n}^{(-1)}[x ; a, b]=\left[g_{i, j}^{(-1)}(x ; a, b)\right]$, $i, j=1, \ldots, n$ which gives a correlation between the generalized Fibonacci matrix $\mathcal{F}_{n}^{(a, b,-1)}$ and the Pascal matrix $\mathcal{P}_{n}^{(-1)}[x]$. 
Example 3.1. The $6 \times 6$ Pascal matrix $\mathcal{P}_{6}^{(-1)}[x]$ is equal to

$$
\mathcal{P}_{6}^{(-1)}[x]=\left(\begin{array}{llllll}
0 & 0 & 0 & 0 & 0 & 0 \\
x & 0 & 0 & 0 & 0 & 0 \\
x^{2} & 2 x & 0 & 0 & 0 & 0 \\
x^{3} & 3 x^{2} & 3 x & 0 & 0 & 0 \\
x^{4} & 4 x^{3} & 6 x^{2} & 4 x & 0 & 0 \\
x^{5} & 5 x^{4} & 10 x^{3} & 10 x^{2} & 5 x & 0
\end{array}\right) .
$$

After we adopt the following two conventions: $0^{0}=1$ and $\left(\begin{array}{l}n \\ k\end{array}\right)=0$ for $k>n$, even in the case $k=0$, we are ready to prove the following statements.

Theorem 3.1. The matrix $\mathcal{G}_{n}^{(-1)}[x ; a, b]=\left[g_{i, j}^{(-1)}(x ; a, b)\right](x \neq 0, a \neq-b)$, whose entries are defined by

$$
g_{i, j}^{(-1)}(x ; a, b)= \begin{cases}x^{-j}\left[\frac{1}{a+b} x^{i+1}\left(\begin{array}{c}
i \\
j-1
\end{array}\right)-\frac{a+2 b}{(a+b)^{2}} x^{i}\left(\begin{array}{c}
i-1 \\
j-1
\end{array}\right)-\right. & \\
\left.\sum_{k=j+1}^{i-1} \frac{(-b)^{i-k-1}\left(a^{2}+a b-b^{2}\right)}{(a+b)^{i-k+2}} x^{k}\left(\begin{array}{c}
k-1 \\
j-1
\end{array}\right)\right], & (i>j, i \neq n) \\
\frac{i}{a+b} x, & (i=j, i \neq n) \\
0, & \text { otherwise }\end{cases}
$$

satisfies

$$
\mathcal{P}_{n}^{(-1)}[x]=\mathcal{F}_{n}^{(a, b,-1)} \mathcal{G}_{n}^{(-1)}[x ; a, b] .
$$

Proof. Let us denote $\mathcal{F}_{n}=\mathcal{F}_{n}^{(a, b,-1)}, \mathcal{F}_{n}^{\dagger}=\mathcal{F}_{n}^{\dagger}(a, b,-1), \mathcal{P}_{n}=\mathcal{P}_{n}^{(-1)}[x]$ and $\mathcal{G}_{n}=\mathcal{G}_{n}^{(-1)}[x ; a, b]$. The following hold:

$$
\begin{aligned}
\mathcal{P}_{n}=\mathcal{F}_{n} \mathcal{G}_{n} & \Longrightarrow \mathcal{F}_{n}^{\dagger} \mathcal{P}_{n}=\mathcal{F}_{n}^{\dagger} \mathcal{F}_{n} \mathcal{G}_{n} \\
& \Longrightarrow \mathcal{F}_{n}^{\dagger} \mathcal{P}_{n}=\mathcal{Z}_{n} \mathcal{G}_{n} .
\end{aligned}
$$

Since all the entries of the last row in matrices $\mathcal{F}_{n}^{\dagger} \mathcal{P}_{n}$ and $\mathcal{Z}_{n} \mathcal{G}_{n}$ are equal to 0 , we have $\mathcal{Z}_{n} \mathcal{G}_{n}=\mathcal{G}_{n}$ and

$$
\mathcal{P}_{n}=\mathcal{F}_{n} \mathcal{G}_{n} \Longrightarrow \mathcal{F}_{n}^{\dagger} \mathcal{P}_{n}=\mathcal{G}_{n}
$$

On the other hand the following is valid:

$$
\begin{aligned}
\mathcal{F}_{n}^{\dagger} \mathcal{P}_{n}=\mathcal{G}_{n} & \Longrightarrow \mathcal{F}_{n} \mathcal{F}_{n}^{\dagger} \mathcal{P}_{n}=\mathcal{F}_{n} \mathcal{G}_{n} \\
& \Longrightarrow \mathcal{V}_{n} \mathcal{P}_{n}=\mathcal{F}_{n} \mathcal{G}_{n} \\
& \Longrightarrow \mathcal{P}_{n}=\mathcal{F}_{n} \mathcal{G}_{n} .
\end{aligned}
$$

Therefore, we get

$$
\mathcal{P}_{n}=\mathcal{F}_{n} \mathcal{G}_{n} \Longleftrightarrow \mathcal{F}_{n}^{\dagger} \mathcal{P}_{n}=\mathcal{G}_{n} .
$$

Hence, it is sufficient to verify

$$
\mathcal{F}_{n}^{\dagger} \mathcal{P}_{n}=\mathcal{G}_{n}
$$


It is clear that the last row in both matrices is equal to 0 . Also, $g_{i, j}=$ $\sum f_{i, k}^{\dagger} p_{k, j}=0$ for $i \leq j-1$. For $i=j$ we have

$$
\left[\mathcal{F}_{n}^{\dagger} \mathcal{P}_{n}\right]_{(i, i)}=f_{i, i+1}^{\dagger} p_{i+1, i}=\frac{1}{a+b} x^{i-i+1}\left(\begin{array}{c}
i \\
i-1
\end{array}\right)=\frac{i}{a+b} x=g_{i, i}
$$

Finally, for $i>j$, applying the result from Theorem 2.1 we have the following

$$
\begin{aligned}
{\left[\mathcal{F}_{n}^{\dagger} \mathcal{P}_{n}[x]\right]_{(i, j)}=} & \sum_{k=j+1}^{i-1} f_{i, k}^{\dagger} p_{k, j}+f_{i, i}^{\dagger} p_{i, j}+f_{i, i+1}^{\dagger} p_{i+1, j} \\
= & -\sum_{k=j+1}^{i-1} \frac{(-b)^{i-k-1}\left(a^{2}+a b-b^{2}\right)}{(a+b)^{i-k+2}} x^{k-j}\left(\begin{array}{c}
k-1 \\
j-1
\end{array}\right) \\
& -\frac{a+2 b}{(a+b)^{2}} x^{i-j}\left(\begin{array}{c}
i-1 \\
j-1
\end{array}\right)+\frac{1}{a+b} x^{i-j+1}\left(\begin{array}{c}
i \\
j-1
\end{array}\right) \\
= & x^{-j}\left[\frac{1}{a+b} x^{i+1}\left(\begin{array}{c}
i \\
j-1
\end{array}\right)-\frac{a+2 b}{(a+b)^{2}} x^{i}\left(\begin{array}{c}
i-1 \\
j-1
\end{array}\right)\right. \\
& \left.-\sum_{k=j+1}^{i-1} \frac{(-b)^{i-k-1}\left(a^{2}+a b-b^{2}\right)}{(a+b)^{i-k+2}} x^{k}\left(\begin{array}{l}
k-1 \\
j-1
\end{array}\right)\right] \\
= & g_{i, j}
\end{aligned}
$$

and the proof is completed.

The theorem above produces a following result in a partial case $a=0, b=1$ and $x=1$.

Corollary 3.1. Let $\mathcal{M}_{n}$ be the matrix with elements defined by

$$
m_{i, j}= \begin{cases}\left(\begin{array}{c}
i \\
j-1
\end{array}\right)-2\left(\begin{array}{c}
i-1 \\
j-1
\end{array}\right)+\sum_{k=j+1}^{i-1}(-1)^{i-k-1}\left(\begin{array}{c}
k-1 \\
j-1
\end{array}\right), & (i>j, i \neq n) \\
i, & (i=j, i \neq n) \\
0, & \text { otherwise }\end{cases}
$$

The Pascal matrix of type -1 and the Fibonacci matrix of type -1 are related with $\mathcal{P}_{n}^{(-1)}=\mathcal{F}_{n}^{(-1)} \mathcal{M}_{n}$.

Proof. The proof follows from $\mathcal{M}_{n}=\mathcal{G}_{n}^{(-1)}[1 ; 0,1]$.

In the case $a=2, b=1$ and $x=1$ from Theorem 3.1 we give a corresponding result for Lucas matrices:

Corollary 3.2. The Pascal matrix and the Lucas matrix satisfy $\mathcal{P}_{n}^{(-1)}=$ $\mathcal{L}_{n}^{(-1)} \mathcal{G}_{n}^{(-1)}[1 ; 2,1]$, where

$g_{i, j}^{(-1)}(1 ; 2,1)= \begin{cases}\frac{1}{3}\left(\begin{array}{c}i \\ j-1\end{array}\right)-\frac{4}{9}\left(\begin{array}{c}i-1 \\ j-1\end{array}\right)-5 \sum_{k=j+1}^{i-1} \frac{(-1)^{i-k-1}}{3^{i-k+2}}\left(\begin{array}{c}k-1 \\ j-1\end{array}\right), & (i>j, i \neq n) \\ \frac{i}{3}, & (i=j, i \neq n) \\ 0, & \text { otherwise. }\end{cases}$ 
Theorem 3.2. The matrix $\mathcal{H}_{n}^{(-1)}[x ; a, b]=\left[h_{i, j}^{(-1)}(x ; a, b)\right](x \neq 0, a \neq-b)$, whose entries are defined by

$$
h_{i, j}^{(-1)}(x ; a, b)= \begin{cases}x^{i}\left[\frac{1}{a+b} x^{-j-1}\left(\begin{array}{c}
i-1 \\
j-2
\end{array}\right)-\frac{a+2 b}{(a+b)^{2}} x^{-j}\left(\begin{array}{c}
i-1 \\
j-1
\end{array}\right)-\right. & \\
\left.\sum_{k=j+1}^{i-1} \frac{(-b)^{k-j-1}\left(a^{2}+a b-b^{2}\right)}{(a+b)^{k-j+2}} x^{-k}\left(\begin{array}{c}
i-1 \\
k-1
\end{array}\right)\right], & (i>j, j \neq 1) \\
\frac{i-1}{a+b} x, & (i=j, j \neq 1) \\
0, & \text { otherwise }\end{cases}
$$

satisfies

$$
\mathcal{P}_{n}^{(-1)}[x]=\mathcal{H}_{n}^{(-1)}[x ; a, b] \mathcal{F}_{n}^{(a, b,-1)} .
$$

Proof. Similarly as in Theorem 3.1 we have that the following equalities are identical

$$
\mathcal{P}_{n}=\mathcal{H}_{n} \mathcal{F}_{n} \Longleftrightarrow \mathcal{P}_{n} \mathcal{F}_{n}^{\dagger}=\mathcal{H}_{n} .
$$

Hence, it is sufficient to verify

$$
\mathcal{P}_{n} \mathcal{F}_{n}^{\dagger}=\mathcal{H}_{n}
$$

The proof can be completed in the same way as in the proof of Theorem 3.1.

The theorem above produces the following result in the partial case $a=0$, $b=1$ and $x=1$.

Corollary 3.3. Let $\mathcal{N}_{n}$ be the matrix with elements defined by

$$
n_{i, j}= \begin{cases}\left(\begin{array}{l}
i-1 \\
j-2
\end{array}\right)-2\left(\begin{array}{l}
i-1 \\
j-1
\end{array}\right)+\sum_{k=j+1}^{i-1}(-1)^{k-j-1}\left(\begin{array}{c}
i-1 \\
k-1
\end{array}\right), & (i>j, j \neq 1) \\
i-1, & (i=j, j \neq 1) \\
0, & \text { otherwise }\end{cases}
$$

The Pascal matrix and the Fibonacci matrix are related with $\mathcal{P}_{n}^{(-1)}=\mathcal{N}_{n} \mathcal{F}_{n}^{(-1)}$.

Proof. The proof follows from $\mathcal{N}_{n}=\mathcal{H}_{n}^{(-1)}[1 ; 0,1]$.

In the case $a=2, b=1$ and $x=1$ from Theorem 3.2 we give a corresponding result for Lucas matrices.

Corollary 3.4. The Pascal matrix and the Lucas matrix satisfy $\mathcal{P}_{n}^{(-1)}=$ $\mathcal{H}_{n}^{(-1)}[1 ; 2,1] \mathcal{L}_{n}^{(-1)}$, where

$h_{i, j}^{(-1)}(1 ; 2,1)= \begin{cases}\frac{1}{3}\left(\begin{array}{c}i-1 \\ j-2\end{array}\right)-\frac{4}{9}\left(\begin{array}{c}i-1 \\ j-1\end{array}\right)-5 \sum_{k=j+1}^{i-1} \frac{(-1)^{k-j-1}}{3^{k-j+2}}\left(\begin{array}{l}i-1 \\ k-1\end{array}\right), & (i>j, j \neq 1) \\ \frac{i-1}{3}, & (i=j, j \neq 1) \\ 0, & \text { otherwise. }\end{cases}$

The generalized Pascal matrix of the second kind and of the type $s$, denoted by $\mathcal{Q}_{n}^{(s)}[x]=\left[q_{i, j}^{(s)}[x]\right], i, j=1, \ldots, n$, is defined by:

$$
q_{i, j}^{(s)}[x]= \begin{cases}x^{i+j-2}\left(\begin{array}{l}
i-1 \\
j-1
\end{array}\right), & i-j+s \geq 0, \\
0, & i-j+s<0 .\end{cases}
$$


Theorem 3.3. The matrices

$$
\mathcal{S}_{n}[x ; a, b]=\left[s_{i, j}(x ; a, b)\right] \text { and } \mathcal{T}_{n}[x, a, b]=\left[t_{i, j}(x ; a, b)\right],
$$

$i, j=1, \ldots, n,(a \neq-b)$ whose entries are defined by

$$
s_{i, j}(x ; a, b)= \begin{cases}x^{j}\left[\frac{1}{a+b} x^{i-1}\left(\begin{array}{c}
i \\
j-1
\end{array}\right)-\frac{a+2 b}{(a+b)^{2}} x^{i-2}\left(\begin{array}{c}
i-1 \\
j-1
\end{array}\right)-\right. & \\
\left.\sum_{k=j+1}^{i-1} \frac{(-b)^{i-k-1}\left(a^{2}+a b-b^{2}\right)}{(a+b)^{i-k+2}} x^{k-2}\left(\begin{array}{c}
k-1 \\
j-1
\end{array}\right)\right], & (i>j, i \neq n) \\
\frac{i}{a+b} x, & (i=j, i \neq n) \\
0, & \text { otherwise }\end{cases}
$$

$$
t_{i, j}(x ; a, b)= \begin{cases}x^{i}\left[\frac{1}{a+b} x^{j-3}\left(\begin{array}{l}
i-1 \\
j-2
\end{array}\right)-\frac{a+2 b}{(a+b)^{2}} x^{j-2}\left(\begin{array}{l}
i-1 \\
j-1
\end{array}\right)-\right. & \\
\left.\sum_{k=j+1}^{i-1} \frac{(-b)^{k-j-1}\left(a^{2}+a b-b^{2}\right)}{(a+b)^{k-j+2}} x^{k-2}\left(\begin{array}{l}
i-1 \\
k-1
\end{array}\right)\right], & (i>j, j \neq 1) \\
\frac{i-1}{a+b} x, & (i=j, j \neq 1) \\
0, & \text { otherwise }\end{cases}
$$

satisfy

$$
\begin{aligned}
\mathcal{Q}_{n}^{(-1)}[x] & =\mathcal{F}_{n}^{(a, b,-1)} \mathcal{S}_{n}[x ; a, b] \\
\mathcal{Q}_{n}^{(-1)}[x] & =\mathcal{T}_{n}[x ; a, b] \mathcal{F}_{n}^{(a, b,-1)}
\end{aligned}
$$

Proof. Similar as the proof of Theorem 3.1.

Corollary 3.5. In the case $a \neq-b$ the matrix $\mathcal{G}_{n}^{(-1)}\left[-\frac{b}{a+b} ; a, b\right]$ is defined by $(3.10)$

$$
g_{i, j}^{(-1)}= \begin{cases}\frac{(-b)^{i-j-1}}{(a+b)^{i-j+2}}\left[b^{2}\left(\begin{array}{c}
i \\
j-1
\end{array}\right)+b(a+2 b)\left(\begin{array}{c}
i-1 \\
j-1
\end{array}\right)-\right. & \\
-\frac{b i}{(a+b)^{2}}, & (i>j, i \neq n) \\
0, & (i=j, i \neq n)\end{cases}
$$

and satisfies

$$
\mathcal{P}_{n}^{(-1)}[-b /(a+b)]=\mathcal{F}_{n}^{(a, b,-1)} \mathcal{G}_{n}^{(-1)}[-b /(a+b) ; a, b] .
$$

Proof. Follows from Theorem 3.1 and the following simple combinatorial identity

$$
\sum_{k=j+1}^{i-1}\left(\begin{array}{l}
k-1 \\
j-1
\end{array}\right)=\left(\begin{array}{c}
i-1 \\
j
\end{array}\right)-1
$$


Corollary 3.6. The matrix $\mathcal{S}_{n}\left[-\frac{b}{a+b} ; a, b\right](a \neq-b)$ is defined by

$$
s_{i, j}= \begin{cases}\frac{(-b)^{i+j-3}}{(a+b)^{i+j}}\left[b^{2}\left(\begin{array}{c}
i \\
j-1
\end{array}\right)+b(a+2 b)\left(\begin{array}{c}
i-1 \\
j-1
\end{array}\right)-\right. & \\
\left.-\left(a^{2}+a b-b^{2}\right)\left(\left(\begin{array}{c}
i-1 \\
j
\end{array}\right)-1\right)\right], & (i>j, i \neq n) \\
-\frac{b i}{(a+b)^{2}}, & (i=j, i \neq n) \\
0, & \text { otherwise }\end{cases}
$$

and satisfies

$$
\mathcal{Q}_{n}^{(-1)}[-b /(a+b)]=\mathcal{F}_{n}^{(a, b,-1)} \mathcal{S}_{n}[-b /(a+b) ; a, b] .
$$

\section{Some combinatorial identities}

In this section we investigate some combinatorial identities involving the generalized Fibonacci numbers.

Theorem 4.1. Let $i, j$ be positive integers satisfying $i \geq j+3$ and $a \neq-b$. The following is valid

$$
\begin{aligned}
& \left(\frac{-b}{a+b}\right)^{i-j}\left(\begin{array}{c}
i-1 \\
j-1
\end{array}\right) \\
= & -F_{i-j+1}^{(a, b)} \frac{b i}{(a+b)^{2}}+F_{i-j}^{(a, b)} \frac{b j}{2(a+b)^{3}}[2 a+(5+j) b] \\
+ & \sum_{k=j+2}^{i-1} F_{i-k+1}^{(a, b)} \frac{(-b)^{k-j-1}}{(a+b)^{k-j+2}}\left[b^{2}\left(\begin{array}{c}
k \\
j-1
\end{array}\right)+b(a+2 b)\left(\begin{array}{c}
k-1 \\
j-1
\end{array}\right)\right. \\
& \left.-\left(a^{2}+a b-b^{2}\right)\left(\left(\begin{array}{c}
k-1 \\
j
\end{array}\right)-1\right)\right] .
\end{aligned}
$$

Proof. From (3.10) we derive the following identities:

$$
\begin{aligned}
g_{j, j}^{(-1)}\left(-\frac{b}{a+b} ; a, b\right) & =-\frac{b i}{(a+b)^{2}}, \\
g_{j+1, j}^{(-1)}\left(-\frac{b}{a+b} ; a, b\right) & =\frac{b}{(a+b)^{3}}\left[b \frac{j(j+1)}{2}+(a+2 b) j\right] \\
& =\frac{b j}{2(a+b)^{3}}[b j+5 b+2 a] \\
& =\frac{b j}{2(a+b)^{3}}[2 a+(5+j) b] .
\end{aligned}
$$

Now, the proof can be derived applying the last, and the following identity

$$
p_{i, j}^{(-1)}\left[-\frac{b}{a+b}\right]= \begin{cases}\left(-\frac{b}{a+b}\right)^{i-j}\left(\begin{array}{l}
i-1 \\
j-1
\end{array}\right), & i-j-1 \geq 0 \\
0, & i-j-1<0\end{cases}
$$

together with (3.10), (3.11) and (1.4). 
Theorem 4.2. If $i, j$ are positive integers satisfying $i \geq j+3$ and $a \neq-b$ we have

$$
\begin{aligned}
& \left(-\frac{b}{a+b}\right)^{i+j-2}\left(\begin{array}{c}
i-1 \\
j-1
\end{array}\right) \\
= & -F_{i-j+1}^{(a, b)} \frac{b i}{(a+b)^{2}}-F_{i-j}^{(a, b)} \frac{b^{2 j-2} j}{2(a+b)^{2 j+1}}[2 a+(5+j) b] \\
+ & \sum_{k=j+2}^{i-1} F_{i-k+1}^{(a, b)} \frac{(-b)^{k+j-3}}{(a+b)^{k+j}}\left[b^{2}\left(\begin{array}{c}
k \\
j-1
\end{array}\right)+b(a+2 b)\left(\begin{array}{c}
k-1 \\
j-1
\end{array}\right)\right. \\
& \left.-\left(a^{2}+a b-b^{2}\right)\left(\left(\begin{array}{c}
k-1 \\
j
\end{array}\right)-1\right)\right] .
\end{aligned}
$$

Proof. From (3.12) we derive the following identities:

$$
\begin{aligned}
s_{j, j}\left(-\frac{b}{a+b} ; a, b\right) & =-\frac{b j}{(a+b)^{2}}, \\
s_{j+1, j}\left(-\frac{b}{a+b} ; a, b\right) & =\frac{-b^{2 j-2}}{(a+b)^{2 j+1}}\left[b \frac{j(j+1)}{2}+(a+2 b) j\right] \\
& =\frac{-b^{2 j-2} j}{2(a+b)^{2 j+1}}[b j+5 b+2 a] \\
& =\frac{-b^{2 j-2} j}{2(a+b)^{2 j+1}}[2 a+(5+j) b] .
\end{aligned}
$$

Now, the proof can be derived using the last identity, following equality

$$
q_{i, j}^{(-1)}\left[-\frac{b}{a+b}\right]= \begin{cases}\left(-\frac{b}{a+b}\right)^{i+j-2}\left(\begin{array}{l}
i-1 \\
j-1
\end{array}\right), & i-j-1 \geq 0 \\
0, & i-j-1<0\end{cases}
$$

as well as (3.12), (3.13) and (1.4).

Theorem 4.3. For $1 \leq r<n$ and $a \neq-b$ we have

(4.1)

$$
\begin{aligned}
\left(\begin{array}{l}
n-1 \\
r-1
\end{array}\right)= & F_{n-r+1}^{(a, b)} \frac{r}{a+b} \\
+\sum_{l=r+1}^{n-1} F_{n-l+1}^{(a, b)} & {\left[\frac{1}{a+b}\left(\begin{array}{c}
l \\
r-1
\end{array}\right)-\frac{a+2 b}{(a+b)^{2}}\left(\begin{array}{c}
l-1 \\
r-1
\end{array}\right)\right.} \\
& \left.\quad-\sum_{k=r+1}^{l-1} \frac{(-b)^{l-k-1}\left(a^{2}+a b-b^{2}\right)}{(a+b)^{l-k+2}}\left(\begin{array}{c}
k-1 \\
r-1
\end{array}\right)\right]
\end{aligned}
$$


Proof. In the partial case $x=1$ from (3.3) we get

$$
g_{i, j}^{(-1)}(1 ; a, b)= \begin{cases}{\left[\frac{1}{a+b}\left(\begin{array}{c}
i \\
j-1
\end{array}\right)-\frac{a+2 b}{(a+b)^{2}}\left(\begin{array}{c}
i-1 \\
j-1
\end{array}\right)-\right.} & \\
\left.\sum_{k=j+1}^{i-1} \frac{(-b)^{i-k-1}\left(a^{2}+a b-b^{2}\right)}{(a+b)^{i-k+2}}\left(\begin{array}{c}
k-1 \\
j-1
\end{array}\right)\right], & (i>j, i \neq n) \\
\frac{i}{a+b}, & (i=j, i \neq n) \\
0, & \text { otherwise. }\end{cases}
$$

Now, the proof follows from

$$
\left(\begin{array}{c}
n-1 \\
r-1
\end{array}\right)=p_{n, r}^{(-1)}=\sum_{l=r}^{n-1} F_{n-l+1}^{(a, b)} \cdot g_{l, r}^{(-1)}(1 ; a, b) .
$$

In the particular case $a=0, b=1$ previous theorem reduces to:

Corollary 4.1. For $1 \leq r<n$

$$
\left(\begin{array}{l}
n-1 \\
r-1
\end{array}\right)=F_{n-r+1}^{(a, b)} \cdot r+\sum_{l=r+1}^{n-1} F_{n-l+1}^{(a, b)}\left[\left(\begin{array}{c}
l \\
r-1
\end{array}\right)-2\left(\begin{array}{c}
l-1 \\
r-1
\end{array}\right)-\sum_{k=r+1}^{l-1}(-b)^{l-k}\left(\begin{array}{c}
k-1 \\
r-1
\end{array}\right)\right] .
$$

Proof. The proof follows directly from Theorem 4.3.

\section{Test matrices for computing pseudoinverse}

In this section we define a set of test matrices for computing the MoorePenrose inverse. These test matrices are generalized Fibonacci matrices $\mathcal{F}_{n}^{(a, b, s)}$, $s=-1$, defined earlier in this paper, and can be considered as a continuation of the previous report in [33].

Pseudoinverse of generalized Fibonacci matrices $\mathcal{F}_{n}^{(a, b, s)}, s=-1$ is defined in $(2.4)$.

Example 5.1. The $6 \times 6$ matrix $\mathcal{F}_{6}^{\dagger(a, b,-1)}$ is equal to

$$
\mathcal{F}_{6}^{\dagger(a, b,-1)}=\left(\begin{array}{cccccc}
0 & \frac{1}{a+b} & 0 & 0 & 0 & 0 \\
0 & -\frac{a+2 b}{(a+b)^{2}} & \frac{1}{a+b} & 0 & 0 & 0 \\
0 & -\frac{\left(a^{2}+a b-b^{2}\right)}{(a+b)^{3}} & -\frac{a+2 b}{(a+b)^{2}} & \frac{1}{a+b} & 0 & 0 \\
0 & \frac{b\left(a^{2}+a b-b^{2}\right)}{(a+b)^{4}} & -\frac{\left(a^{2}+a b-b^{2}\right)}{(a+b)^{3}} & -\frac{a+2 b}{(a+b)^{2}} & \frac{1}{a+b} & 0 \\
0 & -\frac{b^{2}\left(a^{2}+a b-b^{2}\right)}{(a+b)^{5}} & \frac{b\left(a^{2}+a b-b^{2}\right)}{(a+b)^{4}} & -\frac{\left(a^{2}+a b-b^{2}\right)}{(a+b)^{3}} & -\frac{a+2 b}{(a+b)^{2}} & \frac{1}{a+b} \\
0 & 0 & 0 & 0 & 0 & 0
\end{array}\right) .
$$

\section{Conclusion}

Properties, inverse and combinatorial identities for generalized Fibonacci matrices in regular cases $s=0$ and $s=1$ are investigated in [23]. At this moment we consider singular matrices $\mathcal{F}_{n}^{(a, b, s)}, s<0$ with a special view on $s=-1$. Instead of the usual matrix inverse, we use the Moore-Penrose 
pseudoinverse. We compute the pseudoinverse of generalized Fibonacci matrix $\mathcal{F}_{n}^{(a, b,-1)}$. Using these results, a correlation between the matrix $\mathcal{F}_{n}^{(a, b,-1)}$ and the Pascal matrix of the first and the second kind is derived. Later we get some combinatorial identities involving generalized Fibonacci numbers and binomial coefficients. A class of test matrices for computing the Moore-Penrose inverse is given.

\section{References}

[1] V. M. Adukov, Generalized inversion of finite rank Hankel and Toeplitz operators with rational matrix symbols, Linear Algebra Appl. 290 (1999), no. 1-3, 119-134.

[2] R. Aggarwala and M. P. Lamoureux, Inverting the Pascal matrix plus one, Amer. Math. Monthly 109 (2002), no. 4, 371-377.

[3] A. Ashrafi and P. M. Gibson, An involutory Pascal matrix, Linear Algebra Appl. 387 (2004), 277-286.

[4] A. Ben-Israel and T. N. E. Greville, Generalized Inverses, Second Ed., Springer-Verlag, New York, 2003.

[5] G. S. Call and D. J. Velleman, Pascal's matrices, Amer. Math. Monthly 100 (1993), no. $4,372-376$.

[6] R. Chan and M. Ng, Conjugate gradient methods for Toeplitz systems, SIAM Rev. 38 (1996), no. 3, 427-482.

[7] G.-S. Cheon and J.-S. Kim, Stirling matrix via Pascal matrix, Linear Algebra Appl. 329 (2001), no. 1-3, 49-59.

[8] P. Courrieu, Fast Computation of Moore-Penrose Inverse Matrices, Neural Information Processing-Letters and Reviews 8 (2005), no. 2, 25-29.

[9] M. C. Gouveia, Generalized invertibility of Hankel and Toeplitz matrices, Linear Algebra Appl. 193 (1993), 95-106.

[10] U. Grenander and M. Rosenblatt, Statistical Analysis of Stationary Time Series, John Wiley \& Sons, New York; Almqvist \& Wiksell, Stockholm, 1957.

[11] T. N. E. Grevile, Some applications of the pseudoinverse of a matrix, SIAM Rev. 2 (1960), 15-22.

[12] R. E. Hartwig and J. Shoaf, Group inverses and Drazin inverses of bidiagonal and triangular Toeplitz matrices, J. Austral. Math. Soc. Ser. A 24 (1977), no. 1, 10-34.

[13] G. Heinig, Generalized inverses of Hankel and Toeplitz mosaic matrices, Linear Algebra Appl. 216 (1995), 43-59.

[14] G. Heinig and F. Hellinger, On the Bezoutian structure of the Moore-Penrose inverses of Hankel matrices, SIAM J. Matrix Anal. Appl. 14 (1993), no. 3, 629-645.

[15] _ Moore-Penrose inversion of square Toeplitz matrices, SIAM J. Matrix Anal. Appl. 15 (1994), no. 2, 418-450.

[16] A. F. Horadam, A generalized Fibonacci sequence, Amer. Math. Monthly 68 (1961), $455-459$.

[17] T. Kailath and A. Sayed, Displacement structure: theory and applications, SIAM Rev. 37 (1995), no. 3, 297-386.

[18] D. Kalman and R. Mena, The Fibonacci numbers-exposed, Math. Mag. 76 (2003), no. $3,167-181$.

[19] T. Koshy, Fibonacci and Lucas Numbers with Applications, Wiley-Interscience, New York, 2001.

[20] G.-Y. Lee, J.-S. Kim, and S.-G. Lee, Factorizations and eigenvalues of Fibonacci and symmetric Fibonacci matrices, Fibonacci Quart. 40 (2002), no. 3, 203-211.

[21] G.-Y. Lee, J.-S. Kim, and S.-H. Cho, Some combinatorial identities via Fibonacci numbers, Discrete Appl. Math. 130 (2003), no. 3, 527-534. 
[22] P. Stănică, Cholesky factorizations of matrices associated with $r$-order recurrent sequences, Integers 5 (2005), no. 2, A16, 11 pp.

[23] P. Stanimirović, J. Nikolov, and I. Stanimirović, A generalization of Fibonacci and Lucas matrices, Discrete Appl. Math. 156 (2008), no. 14, 2606-2619.

[24] P. S. Stanimirović and M. B. Tasić, Partitioning method for rational and polynomial matrices, Appl. Math. Comput. 155 (2004), no. 1, 137-163.

[25] J. E. Walton and A. F. Horadam, Some further identities for the generalized Fibonacci sequence $H_{n}$, Fibonacci Quart. 12 (1974), 272-280.

[26] G. Wang, Y. Wei, and S. Qiao, Generalized Inverses: Theory and Computations, Science Press, Beijing, 2004

[27] Y. Wei, H. Diao, and M. K. Ng, On Drazin inverse of singular Toeplitz matrix, Appl. Math. Comput. 172 (2006), no. 2, 809-817.

[28] S. Wolfram, The Mathematica Book, 4th ed., Wolfram Media/Cambridge University Press, 1999.

[29] Z. Xu, On Moore-Penrose inverses of Toeplitz matrices, Linear Algebra Appl. 169 (1992), 9-15.

[30] Z. Zhang, The linear algebra of the generalized Pascal matrix, Linear Algebra Appl. 250 (1997), 51-60.

[31] Z. Zhang and J. Wang, Bernoulli matrix and its algebraic properties, Discrete Appl. Math. 154 (2006), no. 11, 1622-1632.

[32] Z. Zhang and Y. Zhang, The Lucas matrix and some combinatorial identities, Indian J. Pure Appl. Math. 38 (2007), no. 5, 457-465.

[33] G. Zielke, Report on test matrices for generalized inverses, Computing 36 (1986), no. $1-2,105-162$.

Marko Miladinović

Department of Mathematics

FACULTY OF SCIENCE

UNIVERSITY OF Niš

VišEgradska 33, 18000 Niš, Serbia

E-mail address: markomiladinovic@gmail.com

Predrag Stanimirović

Department of Mathematics

FaCUlty of SCIENCE

UNIVERSITY OF NIŠ

VišEgradSka 33, 18000 Niš, Serbia

E-mail address: pecko@pmf.ni.ac.yu 